\title{
Molecular Imaging and Precision Medicine, Part II
}

Editor

RATHAN M. SUBRAMANIAM

\section{PET CLINICS}

www.pet.theclinics.com

Consulting Editor

ABASS ALAVI

October 2017 • Volume 12 • Number 4 